\title{
Research on Signal of Field Monitor of 7220A Localizer Beacon Subsystem of ILS
}

\author{
C. Q. Qu \\ East China Regional Air Traffic Management Bureau of Civil Aviation of China, Shanghai, China \\ Email: qcq04@163.com
}

Received 19 October 2015; accepted 26 December 2015; published 29 December 2015

Copyright (C 2015 by author and Scientific Research Publishing Inc.

This work is licensed under the Creative Commons Attribution International License (CC BY). http://creativecommons.org/licenses/by/4.0/

(c) (i) Open Access

\begin{abstract}
From the actual perspective of working principle of localizer beacon subsystem of Instrument Landing System (ILS), consideration of the distance information from localizer antenna to field monitor antenna and wide aperture effect of localizer antenna, broke through the limitation of signals synthesized only far-field (FF), established the near-field (NF) model. The three-dimensional mathematical model of localizer beacon was designed, and the signals at both near-field and far-field were analyzed qualitatively. At the environment of Antenna Fault as well as Antenna Distribution Unit (ADU) phase shifter simulation, the characteristics of near-field and far-field were also compared. The simulation results showed that the model met the requirement of theory of localizer beacon, and the gap between two models was so evident, which resulted from the broken geometric symmetry in NF area. The model could provide valuable theoretical basis for performance evaluation and maintenance of the ILS, and meanwhile, provide reference for the further analysis of localizer beacon.
\end{abstract}

\section{Keywords}

Instrument Landing System (ILS), Localizer Beacon, Near Field, Signal Syntheses

\section{Introduction}

The Instrument Landing System (ILS) had its beginnings in the United States and England during the years 1939 to 1945 [1]. Since then, ILS became the international standard system for approach and landing guidance. ILS was adopted by International Civil Aviation Organization (ICAO) in 1947 and will be in service until at least 2020 [2]. The ILS normally consists of a VHF "Localizer (LOC)" for runway alignment guidance, a UHF "Glide Path (GP)" for elevation guidance and "Marker Beacons (MB)" for providing key checkpoints along the approach. The MB are replaced or supplemented, at some time, by a "Distance Measuring Equipment (DME)" to provide continuous reading of distance. The ILS, in its present 90/150 Hz format, supports lateral guidance by LOC and 
longitudinal guidance by GP. For LOC, it produces a course formed by the intersection of two antenna radiation patterns of equal amplitudes. One pattern is modulated by $90 \mathrm{~Hz}$ and the other by $150 \mathrm{~Hz}$. The Course Line (CL) is the vertical plane where the $90 \mathrm{~Hz}$ and $150 \mathrm{~Hz}$ modulations are equal. The $150 \mathrm{~Hz}$ signal is predominant to the right side of the approach, and the $90 \mathrm{~Hz}$ signal is predominant to the left of the approach.

Many series of Localizer Beacons were produced by antenna manufacturing companies. For example, series3500: 3523B [3], 3524 [4], 3525 [5], 3526 [6] and series-7000: 7212A [7], 7212C [8], 7216A [9], 7216C [10], 7220A [11], and 7220B [12] were produced by Normarc, and single frequency: $1 \mathrm{~F}-8$ moderate-aperture, $1 \mathrm{~F}-12$ medium-aperture, dual frequency: 2F-13 wide-aperture, and 2F-21 ultra wide-aperture (Thales ILS-LOC 411Technical Manual, 2000) [13] were produced by Thales.

The guidance function of ILS depends on spatial signals radiated from ground equipment, and the quality of the signals is up to antenna array and electronic equipment, such as Antenna Distribution Network (ADU). So it is of importance to understand and analyze the field signal in the air, which is radiated by antenna array. Also, it is of advisability to simulate the spatial overlying signal, through which method the spatial electromagnetic environment would be forecast before the flight check. Furthermore, it is of use to make simulation of antenna error or signal distortion; next it can be convenient to find the faulty from which antenna. Based on the signal simulation, operation condition of ILS equipment would be mastered well and more scientific; meanwhile, the maintenance of electronic device would be more appropriate and reliable.

In the paper, the simulated software Matrix Laboratory (MATLAB) was used to analyze the 7220A Localizer Beacon Subsystem of ILS. This software could be used to mathematical modeling, simulate and analyze the dynamical system. Not only could it provide intuitive drawing but also afford integrity and accuracy of the data. To provide valuable reference of performance evaluation and operation maintenance of ILS, the theoretically analysis and mathematical modeling of Localizer Beacon were given.

\section{Result and Discussion}

\subsection{Far Field Monitor}

\subsubsection{Introduction of ADU}

The ADU feeds the antenna elements with the proper amplitude and phase of the CSB (carrier and sideband, a signal amplitude modulated to equal depths by the guidance tones, $150 \mathrm{~Hz}$ and $90 \mathrm{~Hz}$ ) and SBO (sideband only, takes the form of a double sideband, suppressed carrier with the two guidance tones modulated in opposite audio phases) signals. By changing the amplitude of the SBO feeding the ADU, the Course Sector Width is adjusted. The Course Line is determined of mainly the mechanical alignment, but could be adjusted by using a phase shifter inserted at the output of the ADU to one of the antennas. For adjusting Course Line effectively, this antenna feeding the maximum SBO amplitude. Table 1 shows all design data for the antenna system. Amplitude values are given in relative volts, phase in electrical degrees. The phase of SBO with $90^{\circ}$ was realized by $90^{\circ}$ hybrid. All the cables from the transmitter to ADU must be cut with the same length and electrical degrees, antenna cables should also have the same electrical degrees, which have been done by factory. On the base of above premise, the spare signal synthesis would be qualified.

\subsubsection{Signal Synthesis}

It can be found at Figure 1 that the two antenna elements for FF model, when the $\mathrm{R}_{0}$ more than $\mathrm{D} 100$ times, to the receive point "dot $P$ ", the $\mathrm{R}_{1}$ and $\mathrm{R}_{2}$ can be seen as paralleled. The vectors $\mathrm{E}_{1}$ and $\mathrm{E}_{2}$ are $\mathrm{E}_{1}=\mathrm{E}_{0} / \beta_{1}, \mathrm{E}_{2}=$ $\mathrm{E}_{0} / \beta_{2}, \mathrm{E}_{0}$ is its amplitude and $\beta$ is its phase. To the "dot P", the vectors $\mathrm{E}_{1}$ and $\mathrm{E}_{2}$ become $\mathrm{E}_{1}=\mathrm{E}_{0} / \underline{\Phi_{1}}+\alpha \sin \theta, \mathrm{E}_{2}=$ $\mathrm{E}_{0} / \underline{\Phi}_{2}-\alpha \sin \theta$. Where $\Phi$ is the initial phase, $\alpha \sin \theta$ is the phase difference comparative to the original point.

The frequency range of localizer beacon is from 108.1 to $111.975 \mathrm{MHz}$, works in very high frequency (VHF). Choicing the frequency $111.1 \mathrm{MHz}$ for instance, then the corresponding wavelength is $2.7 \mathrm{~m}$.

The electrical length $\alpha=\frac{2 \pi}{\lambda} \cdot D$.

Two antenna elements spaced apart: $\quad \boldsymbol{E}_{t}=2 E_{0} \cos \left(\alpha \sin \theta+\frac{\phi_{1}-\phi_{2}}{2}\right) / \frac{\phi_{1}+\phi_{2}}{2}$.

Two antenna elements fed in phase $\left(0^{\circ}\right)$ spaced $2 \mathrm{D}$ apart: $\boldsymbol{E}_{t}=2 E_{0} \cos (\alpha \sin \theta)$

$$
\boldsymbol{E}_{t}=2 E_{0} \cos (\alpha \sin \theta) / \underline{\phi_{0}} .
$$


Table 1. NORMARC 7220A antenna system signal distribution and antenna elements spacing $\left(\mathrm{DS}=4.0^{\circ}\right)$.

\begin{tabular}{|c|c|c|c|c|c|c|c|c|c|}
\hline $\begin{array}{c}\text { Antenna } \\
\text { No. }\end{array}$ & $\begin{array}{l}\text { Dist from } \\
\text { CL (m) }\end{array}$ & $\begin{array}{c}\text { COU CSB } \\
\text { ampl. (V) }\end{array}$ & $\begin{array}{c}\text { COU CSB } \\
\text { phase }\left(^{\circ}\right)\end{array}$ & $\begin{array}{c}\text { COU SBO } \\
\text { ampl. (V) }\end{array}$ & $\begin{array}{c}\text { COU SBO } \\
\text { phase }\left(^{\circ}\right)\end{array}$ & $\begin{array}{l}\text { CLR CSB } \\
\text { ampl. (V) }\end{array}$ & $\begin{array}{l}\text { CLR CSB } \\
\text { phase }\left(\left(^{\circ}\right)\right.\end{array}$ & $\begin{array}{l}\text { CLR SBO } \\
\text { ampl. (V) }\end{array}$ & $\begin{array}{c}\text { CLR } \\
\text { SBO } \\
\text { phase }\left({ }^{\circ}\right)\end{array}$ \\
\hline 1 & -26.03 & 11 & 0 & 3.29 & -90 & & & & \\
\hline 2 & -22.54 & 15 & 0 & 4.20 & -90 & & & 0.32 & 90 \\
\hline 3 & -19.23 & 29 & 0 & 5.75 & -90 & 5.65 & 180 & 0.32 & 90 \\
\hline 4 & -16.09 & 45 & 0 & 6.59 & -90 & 5.65 & 180 & & \\
\hline 5 & -13.13 & 66 & $\Phi+0$ & 6.66 & $\Phi-90$ & 11.72 & $\Phi+180$ & 0.54 & $\Phi-90$ \\
\hline 6 & -10.34 & 82 & 0 & 6.00 & -90 & 11.72 & 180 & 0.32 & -90 \\
\hline 7 & -7.73 & 93 & 0 & 4.82 & -90 & 32.17 & 180 & 4.32 & -90 \\
\hline 8 & -5.30 & 100 & 0 & 3.38 & -90 & 12.86 & 0 & 4.32 & -90 \\
\hline 9 & -3.04 & 100 & 0 & 1.89 & -90 & 94.60 & 180 & 9.25 & -90 \\
\hline 10 & -0.95 & 93 & 0 & 0.55 & -90 & 204.83 & 0 & 37.52 & -90 \\
\hline 11 & 0.95 & 93 & 0 & 0.55 & 90 & 204.83 & 0 & 37.52 & 90 \\
\hline 12 & 3.04 & 100 & 0 & 1.89 & 90 & 94.60 & 180 & 9.25 & 90 \\
\hline 13 & 5.30 & 100 & 0 & 3.38 & 90 & 12.86 & 0 & 4.32 & 90 \\
\hline 14 & 7.73 & 93 & 0 & 4.82 & 90 & 32.17 & 180 & 4.32 & 90 \\
\hline 15 & 10.34 & 82 & 0 & 6.00 & 90 & 11.72 & 180 & 0.32 & 90 \\
\hline 16 & 13.13 & 66 & 0 & 6.66 & 90 & 11.72 & 180 & 0.54 & 90 \\
\hline 17 & 16.09 & 45 & 0 & 6.59 & 90 & 5.65 & 180 & & \\
\hline 18 & 19.23 & 29 & 0 & 5.75 & 90 & 5.65 & 180 & 0.32 & -90 \\
\hline 19 & 22.54 & 15 & 0 & 4.20 & 90 & & & 0.32 & -90 \\
\hline 20 & 26.03 & 11 & 0 & 3.29 & 90 & & & & \\
\hline
\end{tabular}

Note: The voltage amplitudes are relative to the CSB Course amplitude at antenna 8 . The $\Phi$ at antenna 5 is the phase simulated the PH449 with ADU, making it possible to fine-tune the CL DDM.
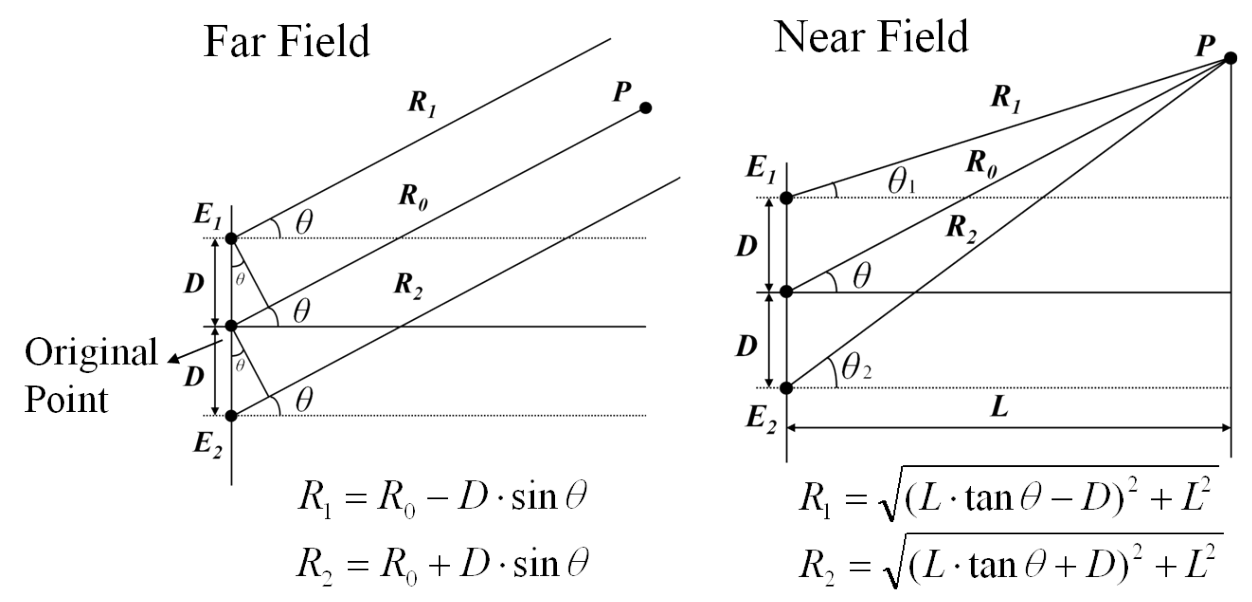

Figure 1. Far Field (FF) model and Near Field (NF) model of two antenna elements. For FF model, the radiation of two antenna elements was parallel. To one receiving point, they have the same azimuth $\theta$. While for NF model, the specific geometry size should be considered, and they had respective azimuth $\theta_{1}$ and $\theta_{2}$. So different from FF model, the calculation method of the distance $R_{1}$ and $R_{2}$ of NF models was not the same, which listing at the bottom of each model. 
Two antenna elements fed out of phase $\left(180^{\circ}\right)$ spaced 2D apart: $\boldsymbol{E}_{t}=2 E_{0} \sin (\alpha \sin \theta)$

$$
\boldsymbol{E}_{t}=2 E_{0} \sin (\alpha \sin \theta) / \underline{\phi_{0}+90^{\circ}} .
$$

From Table 1, the synthesized CSB and SBO can be described as:

$$
C S B=\sum_{n=1}^{10} 2 E_{n} \cos \left(\alpha_{n} \sin \theta\right) / \underline{\phi_{\mathrm{c}}} ; \quad S B O=\sum_{n=1}^{10} 2 E_{n} \sin \left(\alpha_{n} \sin \theta\right) / \underline{\phi_{\mathrm{s}}} .
$$

The DDM is definite as: $D D M=\frac{2 \cdot S B O}{C S B} \cos \left(\phi_{\mathrm{c}}-\phi_{\mathrm{s}}\right)$.

Consider the vertical section, the phase difference consists of two parts: horizontal part $\left(D \cdot 2 \pi / \lambda \cdot \sin \theta_{\mathrm{H}}\right)$ and vertical part $\left(h \cdot 2 \pi / \lambda \cdot \sin \theta_{\mathrm{v}}\right)$.

$$
E_{C S B}=2 \sin \left(D \cdot \frac{2 \pi}{\lambda} \sin \theta_{\mathrm{H}}+h \cdot \frac{2 \pi}{\lambda} \sin \theta_{\mathrm{V}}\right) .
$$

Among them, the distribution function of LPDA is $f(\theta)=10 \frac{-0.0000442|\theta|^{3}-0.0023678|\theta|^{2}-0.0162524|\theta|}{20}$, the fitted function is in accordance with the information supported by antenna designed company. (NORMARC 7000B ILS, 2013) [2] this function is satisfied the azimuth from $-70^{\circ}$ to $+70^{\circ}$, in other words, it can precisely provide the radiation until the distance " $L$ " is less than 10 meter. For example, the LPDA radiation for antenna 1 to the runway center line, the corresponding distance for $70^{\circ}$ is: $L=26.03 \cdot \tan ^{-1} 70^{\circ}=9.47 \mathrm{~m}$.

\subsubsection{Coverage}

Figure 2(a) shows the theoretical Course (COU) and Clearance (CLR) radiation patterns. At the Course Line, the value of CSB reaches maximum while SBO is zero, which realizes the DDM is zero. With the horizontal azimuth $\theta_{\mathrm{H}}$ increasing, the value of CSB slides sharply but SBO shots up. This design makes DDM increasing linear in low azimuth region. The range of the first lobe of SBO is just $0^{\circ}-5^{\circ}$, which reflects the narrow beam. It can avoid multipath reflection causing for obstacle near the both side of the runway. For Course CSB, the peak value of first lobe is greater than the second $40 \mathrm{~dB}$, demonstrating good effect of sidelobe suppression. Besides the main lobe, the coverage task is undertaken by CLR CSB. Again, it is a good design for two frequency capture effect: Each transmitting antenna had its own RF carrier, closely spaced, so that both would be in the passband. The stronger of the two would tend to "capture" the receiver detector [14]. COU realizes direction identification for a narrow azimuth, while CLR mainly works as coverage for a wide angle azimuth. The azimuth exceeding $35^{\circ}$ is not required, so the coverage range is just considered under $40^{\circ}$, the specific coverage requirement [15] is represented in Figure 2(b).

The capture effect is showed in Figure 3, the antenna has its own RF carrier, closely spaced, so that both would be in the passband. The stronger of the two would tend to "capture" the receiver detector. But somewhat dependent on receiver type, such as: linear receiver model, digital receive model and analog receiver model, the capture effect produces, roughly, an audio ratio equal to twice the square of the RF ratio. Thus, there exists a very rapid transition from clearance signal to guidance signal as the azimuth angle changes through the point of equality, the red point in the top of Figure 3. In this paper, the linear receiver model is chose to simulate DDM and SDM curve. The bottom of Figure 3 shows the DDM pattern of COU and CLR respectively. The value of COU DDM -0.155 to 0.155 was defined as DS. This range of the curve has good linear feature, the value of DDM is proportional to the $\theta_{\mathrm{H}}$.

Besides the transmit power, distribution relationship of ADU, the coverage strength also affected by the height of the transmit antennas. Figure 4 shows the vertical pattern of imaging synthesis with horizontal polarization. For a certain height, the field strength of CSB enhanced linearly with increasing of $\theta_{\mathrm{V}}$ below $5^{\circ}$. Obviously, field strength enhanced with increasing of antennas height, almost linear increase below $4 \mathrm{~m}$. Because the radiation lobe made up of antenna and its image was drove down by the antenna height raised, when the height reaches $\lambda /\left(4 \sin \theta_{v}\right)$, the maximum value of lobe was achieved. The red dot line in Figure 4 shows the relative maximum value of CSB, in this case, the height varied with different $\theta_{\mathrm{v}}$, not the constant value. So, in case 


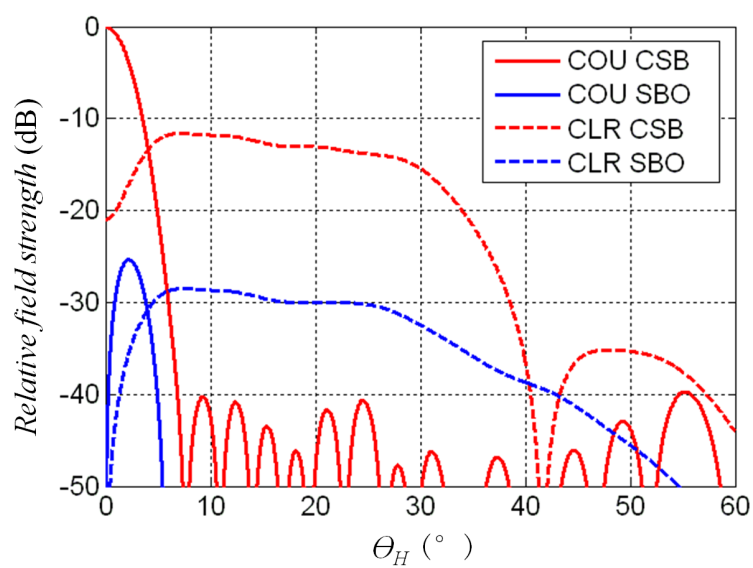

(a)

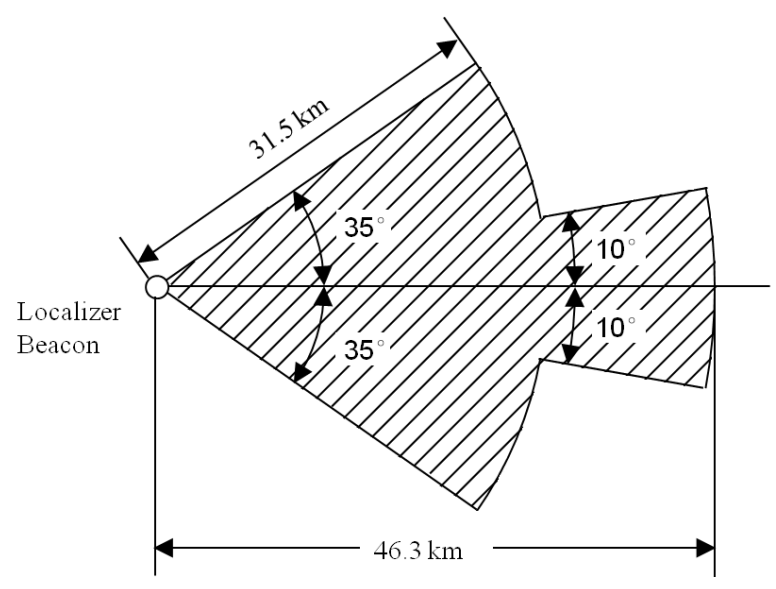

(b)

Figure 2. (a) (Color Online) Theoretical radiation pattern of CSB and SBO distribution at far field with one half of the symmetrical patterns. The height of the antenna is $3 \mathrm{~m}$ and the elevation angle $\theta_{\mathrm{V}}$ is $3^{\circ}$. It can be seen that the horizontal Azimuth $\theta_{\mathrm{H}}$ dependence of relative field strength with four radiation patterns: Course CSB, Course SBO, Clearance CSB and Clearance SBO. The CSB is used to signal coverage with the higher level, while SBO is used to direction identification by its 90 and $150 \mathrm{~Hz}$. The level of Clearance SBO is lower than that plotted in 7220 A instruction manual; (b) Horizontal coverage ruled by Annex 10 (Annex 10, 2006) [15], where a distance of at least $46.3 \mathrm{~km}$ within $\pm 10^{\circ}$ from the course line, and the limits reduced to $31.5 \mathrm{~km}$ between $10^{\circ}-35^{\circ}$ from the front course line. The minimum field in this region must be more than 40 $\mu \mathrm{V} / \mathrm{m}$.
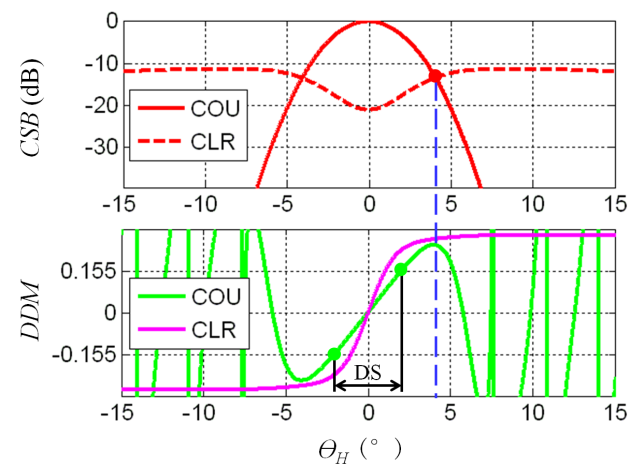

Figure 3. (Color Online) Horizontal Azimuth $\theta_{\mathrm{H}}$ dependence of theoretical CSB and DDM distribution. The top diagram shows the cross point of the level of COU and CLR. at about $4^{\circ}$, the COU level is identical to the CLR level, which could decide the type of DDM, by COU or CLR.

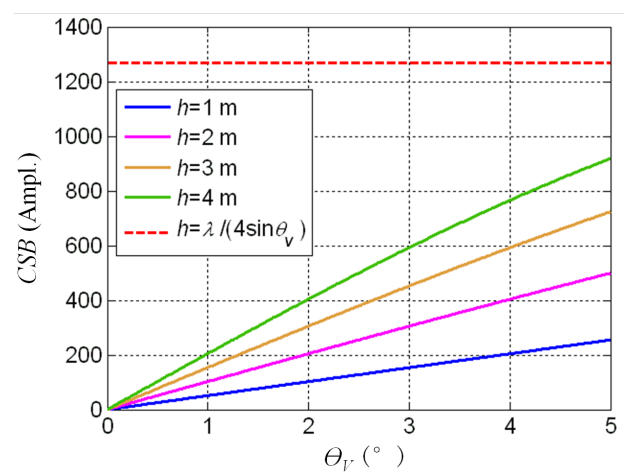

Figure 4. (Color Online) Vertical elevation $\theta_{\mathrm{v}}$ dependence of CSB level with different antennas height. It can be seen that the higher of antennas, the more field strength. And for one antennas height, the more elevation, the more field strength. When the height agree to $\lambda /\left(4 \sin \theta_{v}\right)$, the maximum field strength appears. Because the height is changed by different elevation, the field strength was always the same. 
of low field strength coverage, or coverage not enough, elevate the height of antenna is a advisable choice, which can strengthen the signal coverage effectively. For amplitude of CSB, there is no obvious difference between NF and FF, because the height of antenna is far less than the distance from antenna to receive point. Comparatively the longitudinal distance of antenna is approximate to the distance of NF monitor. So the horizontal differences should be paid more attention to.

The space pattern of CSB coverage is exhibited in Figure 5. It can be seen that the COU signal is far more than that of CLR. This design feature could achieve a good BBP to resist obstruction, and to avoid the influence of reflecting and multipath effect. Also, the CLR signal is quickly reduced outside $\pm 40^{\circ}$, just a "small tail" in $40^{\circ}$ $60^{\circ}$. Making full use and consideration of coverage extend from $-35^{\circ}-35^{\circ}$. [ANNEX 10, (2006)] [15].

\subsubsection{DDM Pattern}

From the vector distribution of CSB and SBO, the DDM and SDM pattern are calculated, shown in Figure 6.

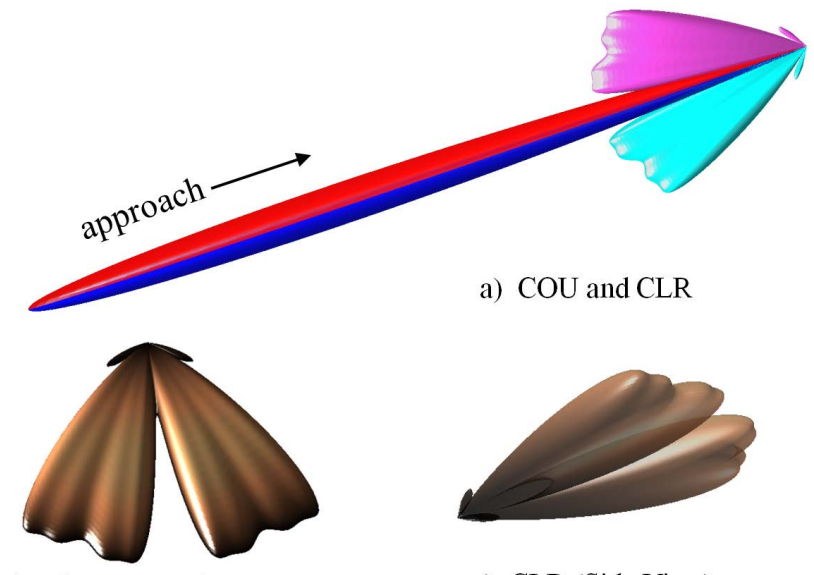

b) CLR (Top View)

c) CLR (Side View)

Figure 5. (Color Online) Three-dimensional FF model of CSB coverage. (a) shows the whole shape, pink (CLR) and red (COU) color indicate the $90 \mathrm{~Hz}$ is dominant and cyan (CLR) and blue (COU) color meaning the $150 \mathrm{~Hz}$ is guiding. (b) and (c) choice the CLR signal to present the space characteristic. All the three pictures just describe the first vertical lobe (elevation from $0^{\circ}$ to $27^{\circ}$ with $\mathrm{h}=3 \mathrm{~m}$ ) and the azimuth is given from $-70^{\circ}$ to $70^{\circ}$. Where (a) and (c) are given perspective effect for achieving senses of the space signal distribution.

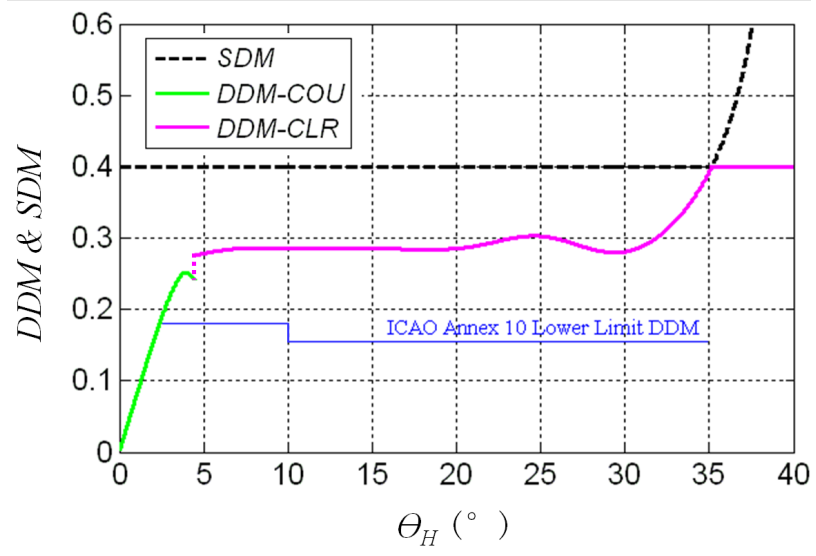

Figure 6. (Color Online) Theoretical DDM and SDM distribution formed by Linear Receiver model with one half of the symmetrical patterns. The performance of the antenna system can be expressed as far field signal patterns. DDM is defined as: $D D M=\mathrm{m}_{150}-\mathrm{m}_{90}$ for modulation level $<2 \mathrm{~m}, D D M=2 \mathrm{~m}$ for modulation level $>2 \mathrm{~m}$; SDM is defined as: $S D M=2 \mathrm{~m}$ for modulation level $<2 \mathrm{~m}, S D M=\mathrm{m}_{150}+\mathrm{m}_{90}$ for modulation level $>2 \mathrm{~m}$. The black dotted line shown in the figure is the SDM, while the green line (CL) and pink line (CLR) represent the DDM. From the angle where the DDM is 0.18 to 10 degrees of the course line, the DDM should not be less than 0.18 . From 10 degrees to 35 degrees the DDM shall not be less than 0.155 , which defined by ICAO Annex 10. The type of DDM is decided by the cross point described in Figure 3. 
The low angle is dominant by course signal, which has a good linear characteristic below $2^{\circ}$. The DDM starts to transition to clearance signal near cross point, where the course and clearance powers are equal. Different to course DDM, the curve of clearance DDM is comparatively smoothly, the value keeps on about 0.3 from $5^{\circ}$ $30^{\circ}$, but steeply rising in the later region. This perfectly satisfies the requirement that from the angle where the DDM is 0.18 to 10 degrees of the course line, the DDM should not be less than 0.18 . From 10 degrees to 35 degrees the DDM shall not be less than 0.155, which defined by ICAO Annex 10 [15].

\subsection{Near Field Monitor}

The position of the FF monitor has been performed at Figure 7(a). Where the value of DS is $D S=2 \cdot \arctan \frac{105}{300+3700}=3.007^{\circ}$. In general, the site of NF monitor is located $60-100 \mathrm{~m}$ away (Normarc 7220A Instruction manual, 2012) [11]. However, the following discussion considers this distance is too close to the antenna, $120 \mathrm{~m}$ is chosen for the NF monitor distance is advisable, kindly find the details in the discussion of DDM pattern. The boundary between NF and FF is the distance: 100 times of D (from Ant.1 to the original point). The region less than this distance is divided to NF, in this case, the FF paralleled model does not suit any more. The NF model has its own characteristic.

\subsubsection{Signal Synthesis}

It can be found at Figure 1 that the two antenna elements for NF model, different from FF model, when $R_{0}<$ 100D, $R_{1}$ and $R_{2}$ could not be seen as paralleled, the specific size should be considered. The value of $R_{1}$ and $R_{2}$ has been given at the bottom of the Figure 1, which is calculated by trigonometric function (the Pythagorean theorem). The three-dimensional coordinate system has been used to establish the 3D model. Original point positioned at the intersection between the runway centerline and the antenna. The $\mathrm{x}$-axis is oriented perpendicular to the runway centerline in the horizontal plane containing the original point. The y-axis is oriented along the runway centerline in the horizontal plane containing the original point. The $z$-axis is perpendicular to both the $\mathrm{x}$-axis and the $\mathrm{y}$-axis, mainly describes the height of antenna and receiving point. The radiation distance, $R$, from one antenna to the receive point is:

$$
R=\sqrt{(L \cdot \tan \theta+D)^{2}+L^{2}+h^{2}} .
$$

$L$ is the distance alone the y-axis, $D$ is the distance alone the x-axis from original point to the antenna, which can found is Table 1 , with its positive value and negative value relative to original point, and h is decided by the vertical anger and the height of the antenna. Different to the $R$ calculated in FF, which is a relative value.

The amplitude of $R_{1}$ and $R_{2}$ is not identical, which is inversely proportional to the radiation distance. So, under the NF circumstance, all the calculation of CSB, SBO, ddm and so on is made by vector synthesis. The synthesis amplitude and phase is:

$$
\begin{gathered}
E_{t}=\sqrt{\left(E_{1} / R_{1}\right)^{2}+\left(E_{2} / R_{2}\right)^{2}+\cdots+\left(E_{n} / R_{n}\right)^{2}} \\
\phi_{t}=\arctan \frac{\sin \left(\phi_{1}-R_{1} \frac{2 \pi}{\lambda}\right) \cdot E_{1} / R_{1}+\sin \left(\phi_{2}-R_{2} \frac{2 \pi}{\lambda}\right) \cdot E_{2} / R_{2}+\cdots+\sin \left(\phi_{n}-R_{n} \frac{2 \pi}{\lambda}\right) \cdot E_{n} / R_{n}}{\cos \left(\phi_{1}-R_{1} \frac{2 \pi}{\lambda}\right) \cdot E_{1} / R_{1}+\cos \left(\phi_{2}-R_{2} \frac{2 \pi}{\lambda}\right) \cdot E_{2} / R_{2}+\cdots+\cos \left(\phi_{n}-R_{n} \frac{2 \pi}{\lambda}\right) \cdot E_{n} / R_{n}} .
\end{gathered}
$$

\subsubsection{Coverage}

Comparing to the pattern of FF, there are several differences. The theoretical Course and Clearance radiation patterns $120 \mathrm{~m}$ far from the original point have been shown in Figure 8. Firstly, relative to the course CSB, the clearance lines are elevated about $10 \mathrm{~dB}$ in lower angle for CSB and $5 \mathrm{~dB}$ for SBO. Secondly, the first lobe of course CSB and course SBO is broaden. The peak of SBO moves from $2.5^{\circ}$ with FF to $6.5^{\circ}$ with NF, and for $\mathrm{CSB}$, the point of $-20 \mathrm{~dB}$ moves from $5^{\circ}$ with FF to $12^{\circ}$ with NF. In a word, the narrow beam of 20 unit wide aperture has been collapsed at near field region. 


\subsubsection{DDM Pattern}

The DDM pattern of NF has been showed in Figure 9. It can be seen that there is no obvious difference for high angle distribution, but for low angle, less than $10^{\circ}$, there are three distinct diversities. One is the degenerating of the linearity, the linear distortion appears in low angle range. Another is the value of course DDM, far less than that of FF. At the position of $4^{\circ}$, DDM is about 0.03 for NF, while it is about $2.4^{\circ}$ for FF, deflates 8 times. Besides, the cross point moves to the higher angle, from $4.1^{\circ}$ to $7.5^{\circ}$. The first difference would be discussed following Figure 10 and the other two analyzed in part 2.3.

There are four representative kind of DDM pattern listed in Figure 10, namely distance of $60 \mathrm{~m}, 80 \mathrm{~m}, 100 \mathrm{~m}$ and $120 \mathrm{~m}$. For DDM with $120 \mathrm{~m}$, the value is increasing monotonically with better linearity. When the distance is $100 \mathrm{~m}$, the linearity becomes worse. Even more, the reversed phase appears at $80 \mathrm{~m}$. The more complex condition happened at $60 \mathrm{~m}$, linearity disappears completely and the DDM curve becomes wave like. Although the linearity of curve at $100 \mathrm{~m}$ is better than that of $60 \mathrm{~m}$, the linearity is so sensitive to the distance within $100 \mathrm{~m}$. Next step the distance dependence of DDM would be discussed. In the conclusion of discussed above, the distance of $120 \mathrm{~m}$ is more suitable for NF monitor site.

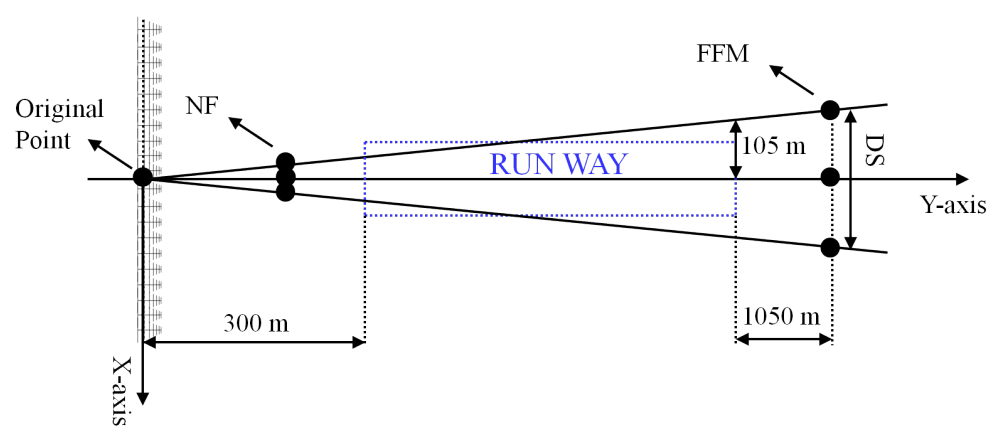

(a)

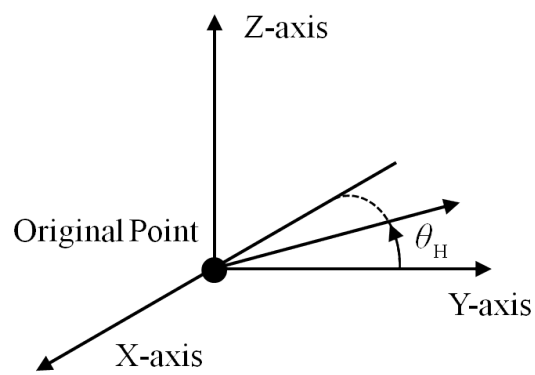

(b)

Figure 7. (Color Online) (a) Top view: The site of run way, transmitter antennas, NF monitor antennas and FFM antennas, where the size is not given in proportion. Supposing the length of run way is $3700 \mathrm{~m}$, then the DS is about $3^{\circ}$. The half width is about $132 \mathrm{~m}$ at FF and about $3 \mathrm{~m}$ at NF (corresponding the distance is $120 \mathrm{~m}$ from NF to the original point). (b) Space pattern: the definition of the horizon and vertical direction. In horizon plane XOY, the direction of rotation angle $\theta_{\mathrm{H}}$, is anticlockwise, which is identical to the definition in Figure 1.

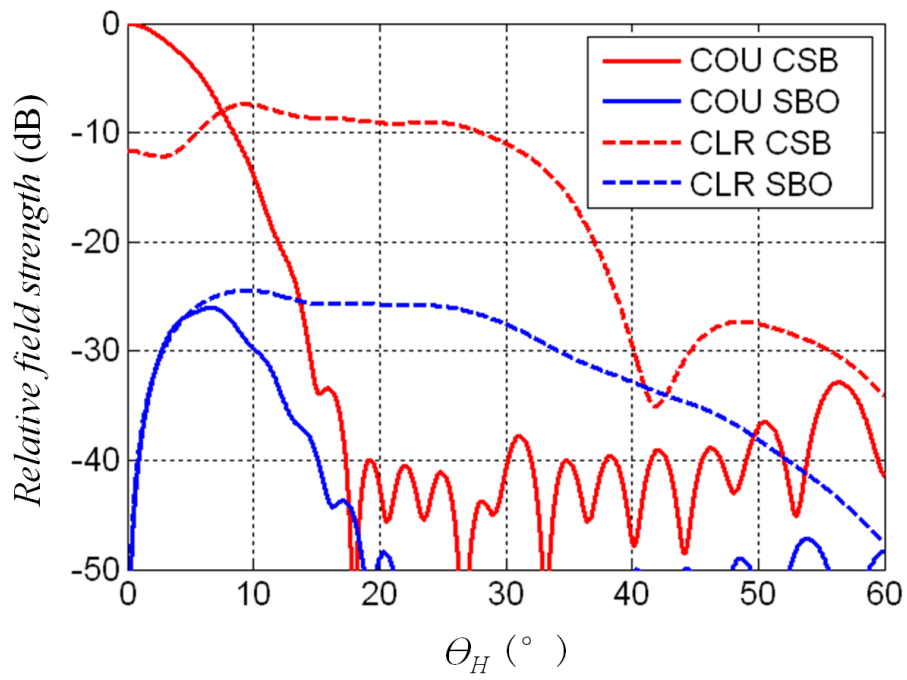

Figure 8. (Color Online) Theoretical radiation pattern of CSB an d SBO distribution at near field (120 m far from the original point) with one half of the symmetrical patterns. The height of the antenna is $3 \mathrm{~m}$ and the elevation angle $\theta_{\mathrm{V}}$ is $3^{\circ}$. It can be seen that the horizontal Azimuth $\theta_{\mathrm{H}}$ dependence of relative field strength with four radiation patterns: Course CSB, Course SBO, Clearance CSB and Clearance SBO. The CSB is used to signal coverage with the higher level, while SBO is used to direction identification by its 90 and $150 \mathrm{~Hz}$. Compare to the diagram of far field, the SBO beams becomes wider. 


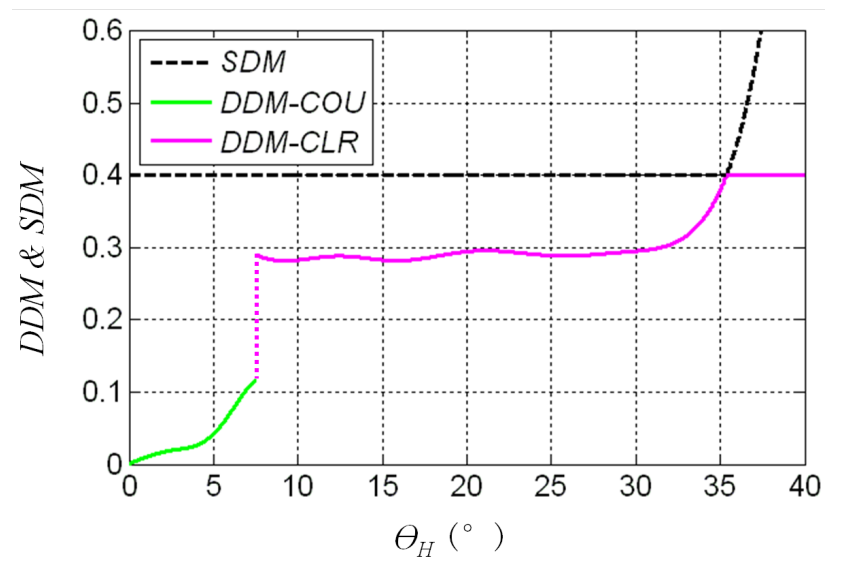

Figure 9. (Color Online) Theoretical DDM and SDM distribution made by Linear Receiver model with one half of the symmetrical patterns. The performance of the antenna system can be expressed as near field signal patterns (120 m far from the original point). Compare to the diagram at far field, the CSB cross point of COU and CLR move to higher degree: from $4^{\circ}$ to $7.5^{\circ}$. Meanwhile, the value of ddm-COU becomes less. Near the course line (CL), the ddm value is far less than that of far field.

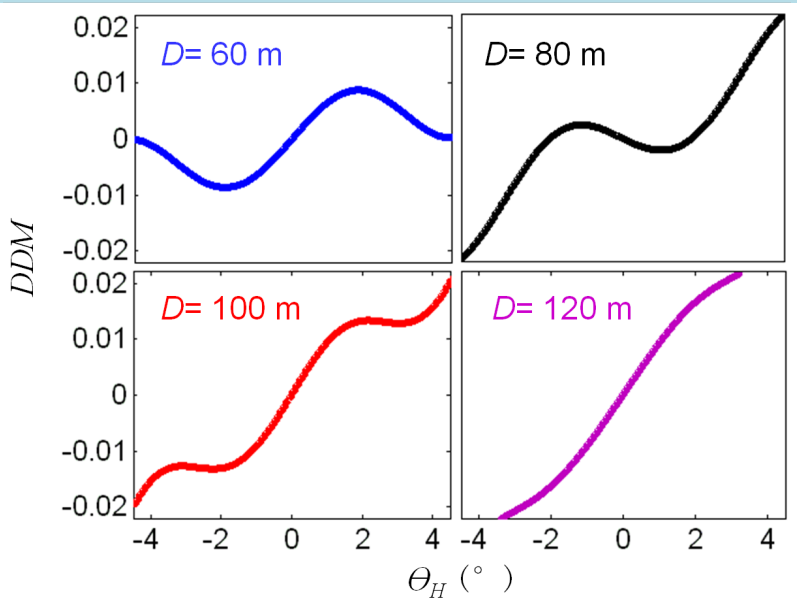

Figure 10. (Color Online) The horizontal azimuth dependence of ddm with different distance. For ddm with $120 \mathrm{~m}$, the value is increasing monotonically with better linearity. When the distance is $100 \mathrm{~m}$, the linearity becomes worse. Even more, the reversed phase appears at $80 \mathrm{~m}$. The more complex condition happened at $60 \mathrm{~m}$, linearity disappears completely and the ddm curve becomes vibrating.

Figure 11 shows the DDM pattern with its distance at different horizontal azimuth $\theta_{\mathrm{H}}$. The DDM value fluctuates below $100 \mathrm{~m}$, and shots up sharply until $1000 \mathrm{~m}$, and then increases mildly, keeps about 0.155 in $\mathrm{FF}$ at last. The enlarged area with four curves of half DS sector up to $4^{\circ}$, seen in the inset, illustrates that the DDM value is enhanced with the DS rising. It is similar to the FF behavior, DDM value enhanced linearly with the increasing of horizontal azimuth, seen in Figure 6. But there is a abnormal behavior near the transmit antenna, the DDM value is fluctuating around the zero point within $80 \mathrm{~m}$, which is result from the rapid changing of phase error at NF. The instruct manual (Normarc 7220 A Instruction manual, 2012) [11] indicates the NF monitor should be sited at $80 \mathrm{~m}$. From what author can see, it is not reasonable. From the inset, the vertical line drew at $80 \mathrm{~m}$, the value of half DS up to $2^{\circ}$ is almost zero, also the reversed phase discussed in Figure 10. So the NF monitor sited at $80 \mathrm{~m}$ is not representative, $120 \mathrm{~m}$ is more suitable. At this distance, the DDM value would not transit to negative anymore, and possesses a better linearity.

\subsection{Comparison of Two Models}

To understand the characteristic of the two models entirely and systemically, the calculation of signal coverage, cross point, DDM pattern as well as simulation of antenna failed and phase shifter have been further researched. 


\subsubsection{Coverage}

The strength of the receive signal decided by two part, radiation loss and antenna gain. The concrete calculation of signal strength at NF and FF monitor position given in following discussion.

$$
\begin{gathered}
\operatorname{Loss}(\mathrm{dB})=32.44+20 \lg f(\mathrm{MHz})+20 \lg D(\mathrm{Km}) \\
\operatorname{Gain}(\mathrm{dB})=G_{t}+G_{r}+G_{\text {ant-array }}+G_{\text {lobing }} \\
G_{\text {ant-array }}=10 \lg \frac{\left(\sum E_{n}\right)^{2}}{\sum E_{n}^{2}} ; G_{\text {lobing }}=20 \lg \left[2 E \sin \left(h \frac{2 \pi}{\lambda} \sin \theta_{\mathrm{V}}\right)\right]
\end{gathered}
$$

where the $G_{t}$ is the gain of single transmitting antenna, for LPDA, $G_{t}$ is about $10 \mathrm{dBi}$.

$G_{r}$ is the gain of monitor antennas, $G_{t}$ of NF and FFM is about $6.5 \mathrm{dBi}$.

FFM: $-7 \mathrm{~dB}, \theta_{\mathrm{V}}=0.069^{\circ}$ for $h=6.0 \mathrm{~m}, D=5 \mathrm{Km}$.

$\mathrm{NF}: 20 \mathrm{~dB}, \theta_{\mathrm{V}}=0.716^{\circ}$ for $h=1.5 \mathrm{~m}, D=120 \mathrm{~m}$.

The difference of loss between FFM and NF is distance, height of antenna and $G_{\text {lobing. }}$.

The $G_{\text {lobing }}$ of NF is $-21.2 \mathrm{~dB}$, and of FFM is $-29.5 \mathrm{~dB}$ (supposing $E=1$ ). The Loss of NF is $54.9 \mathrm{~dB}$, and of FFM is $87.3 \mathrm{~dB}$. So the strength difference between NF and FFM is $(87.3-54.9)+(29.5-21.2)=40.7 \mathrm{~dB}$ in all.

Through the coverage for FF in Figure 2(a) and for NF in Figure 8, it reveals that the cross point of course CSB and clearance CSB is changeable with the distance to original point. It seems that the near one accompanies with the wider degree. The relationship between concrete distance from $50 \mathrm{~m}$ to $4000 \mathrm{~m}$ and their cross point has been shown in Figure 12. The cross point of Azimuth is about $4.1^{\circ}$ at FF (more than 100 times of D: $2600 \mathrm{~m}$ ), gradually move to high angle with the decrease of distance. The cross point reaches to $4.2^{\circ}$ at $1000 \mathrm{~m}$ away, after that, the value rise more evident. It runs up to $5^{\circ}$ at $300 \mathrm{~m}$ away, and shots up to $8^{\circ}$ at $100 \mathrm{~m}$ away. However, for the position of NF monitor listed in Figure 7 , the half DS only $2^{\circ}$, far less than $8^{\circ}$, so course signal is predominant to the position of the three NF monitor antennas.

Figure 13 presents the data tested away from 300 m by NORMARC 7710 [16] Analyzer, whose demodulation mode based on digital signal processing. There are three kinds of signal: the antenna radiated course signal only, radiated clearance signal only and radiated course and clearance simultaneously. Through this testing, the position of cross-point could be detected and the receiving behavior at this point also be researched. For theoretical analysis, the equality point located at $5^{\circ}$, found at Figure 12. In reality, there transition point from course signal to clearance signal also appears at about $5^{\circ}$, which is satisfied the theory well. In this region, the strength of "COU + CRL" is about $2 \mathrm{~dB}$ more than that of transmitting sole signal. This demonstrates both of two signal

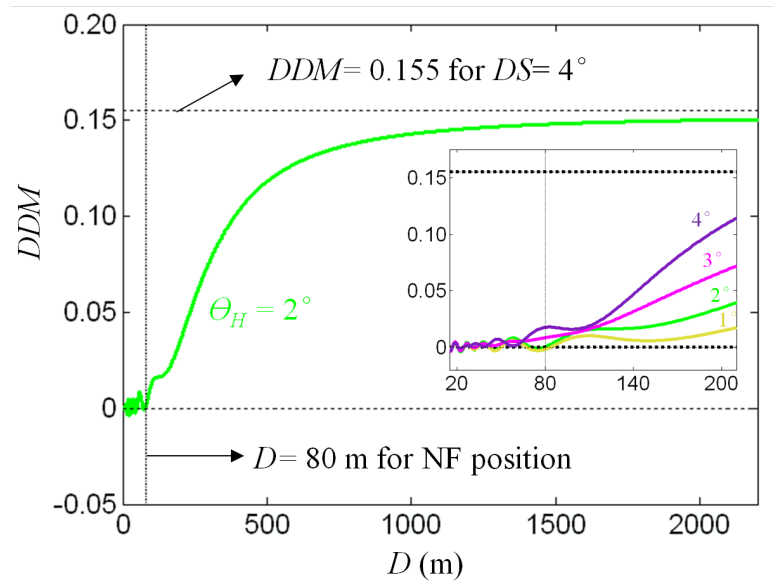

Figure 11. (Color Online) The distance dependence of ddm at different horizontal azimuth with one half of the symmetrical patterns. The ddm value shots up with the increasing of distance at near field, and the value rise smoothly $1000 \mathrm{~m}$ away, close to 0.155 at far field. This is the course sector (CS, or called DS). The inset shows the half DS patterns up to $4^{\circ}$. It can be found that the ddm value is enhanced with the DS rising beyond $100 \mathrm{~m}$ away. In the range of $60 \mathrm{~m}$, the ddm curve is out of order, fluctuating around the zero point. 


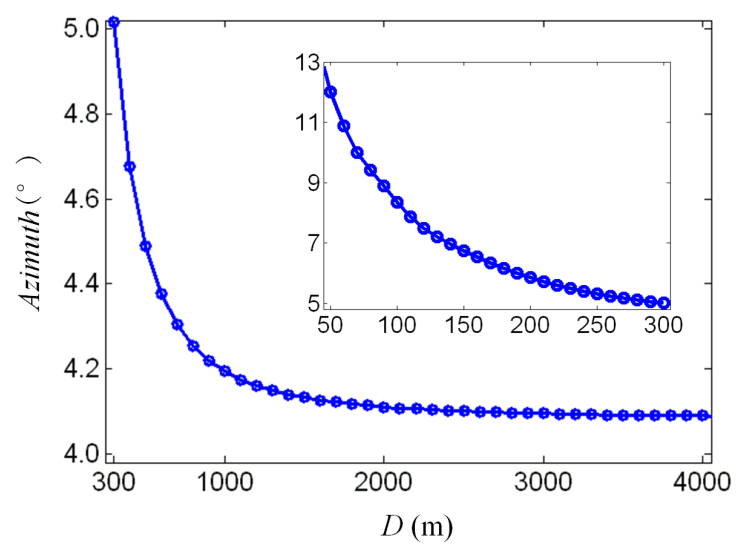

Figure 12. (Color Online) Distance dependence of cross point of CSB-COU and CSB-CLR from $300 \mathrm{~m}$ to $4000 \mathrm{~m}$. The insets show the range of $50 \mathrm{~m}$ to $300 \mathrm{~m}$. Evident, the cross point of azimuth moves to the center line with the increasing of distance. The cross point is about $4.1^{\circ} 3000 \mathrm{~m}$ away, which is indicated in the former Figure 3.

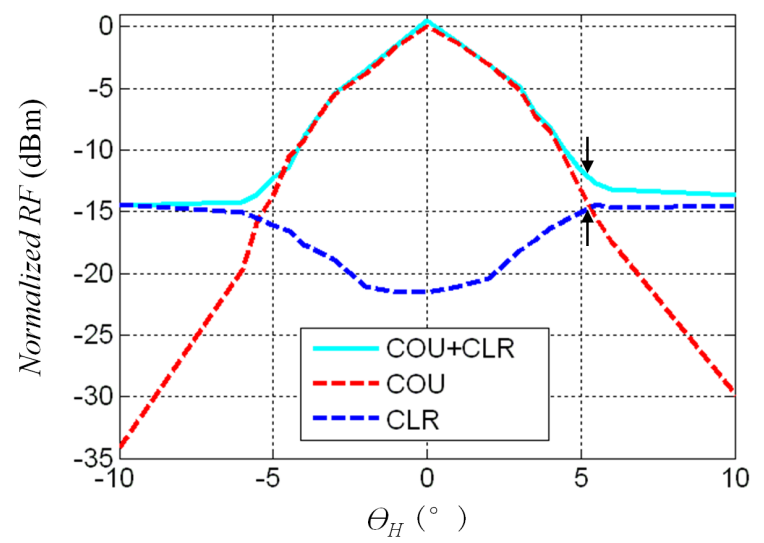

Figure 13. (Color Online) The horizontal azimuth dependence of normalized radio frequency level for transmitting both COU and CLR, COU only and CLR only. Where the value COU transmitted only with $\theta_{\mathrm{H}}=0$ is normalized. The data was tested $300 \mathrm{~m}$ away from the antenna by NORMARC 7710 NAV ANALYZER [16]. The NAV Analyzer uses a digital radio with demodulation based on digital signal processing. In theoretical, the cross point of COU and CLR is located at the position about $5^{\circ}$, the actual value is a little more than $5^{\circ}$ marked in the figure, the value of transmitting dual signal simultaneously is about $2 \mathrm{~dB}$ more than transmitting sole signal. This can be explained by capture effect and digital signal processing model of the NAV analyzer. It is worth mentioning that for the center line position, the value of transmitting dual signal is also little higher than the value of COU signal.

components have been received at the transition region. The discussion has also been mentioned above, described about Figure 3, the type of receiver. Within the scope of $\pm 4^{\circ}$ the "COU" curve almost coincides with the "COU + CRL" curve, except for the position at $0^{\circ}$, the "COU + CRL" signal is little higher than that of "COU". In general, according to the filtering mode, the more strength gap between two signals, the more stable displaying for the higher one. The phenomenon may be result from the measurement error, also from the interference in transmitter.

\subsubsection{DDM Pattern}

Figure 14 lists five DDM curve, four of NF and one of FF. Obviously, the DDM value of NF is far less than that of FF. Although the distribution of four NF ones are different, seen in Figure 10, compare to FF one, they are almost the same behavior and close to the zero point. So, for NF monitor, the monitor value should be adjust first, and then monitor various parameters correctly.

\subsubsection{Antenna Failed Simulation}

The signal failed of some antenna would affect the signal synthesis of both CSB and SBO, thereby changing the 
DDM distribution. The DDM patterns of FF (12,000 m) and NF (120 m) under the simulation circumstance of Ant. 10 failed has been showed in Figure 15. Firstly, to make the two models manifesting the same DS, the DDM curve of NF has been adjusted to 0.155 corresponding to the $\theta_{\mathrm{H}}= \pm 2^{\circ}$. It can be seen the dotted line is the normal DDM curve, the curve of FF reflects the better linear characteristics. Under the Ant. 10 failed circumstance, both the DS of two models has changed. The FF one shortens a little but the NF one decreases to about $3^{\circ}$. Moreover the CL position of NF one has also deviated, seen in the inset. To conclusion, there are two obvious differences under the circumstance of antenna failed.

\subsubsection{Phase Shifter Simulation}

At the flight check situation, sometimes the CL is deviated from the center line, which should be straightened. The phase calibration of shifter simulated with $360^{\circ}$ rotation, set in Ant. 5 with the maximum level of SBO, found in Table 1. Seen in Figure 16, the top one shows the result 12,000 m and $1200 \mathrm{~m}$, the former exhibits the

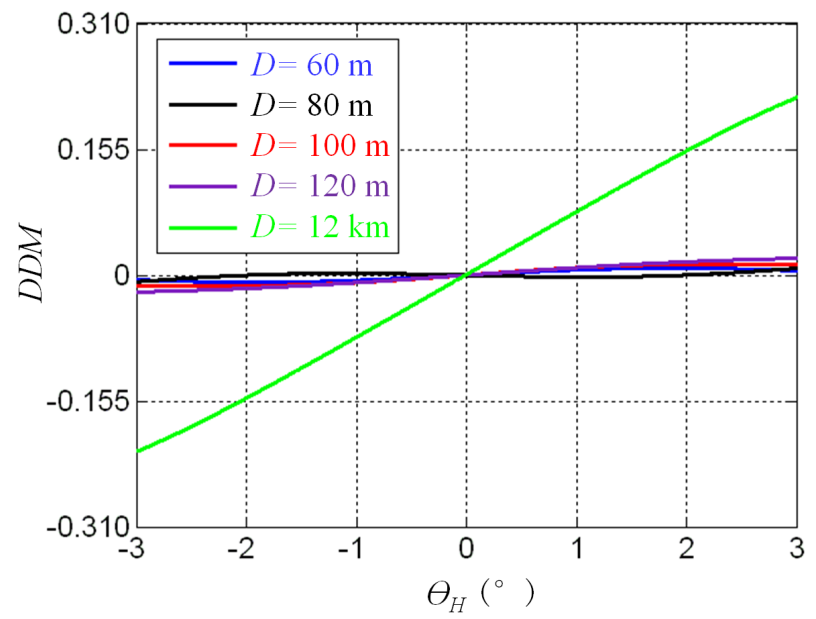

Figure 14. (Color Online) The horizontal azimuth dependence of ddm with different distance: NF for $60 \mathrm{~m}, 80 \mathrm{~m}, 100 \mathrm{~m}$, $120 \mathrm{~m}$, and FF for $12 \mathrm{Km}$. The three dimensional model with the height of antenna is 3 meters, and the elevate angle is 3 degrees. At the near field, the ddm curve exhibits the good linear behavior, and reaches 0.155 at the azimuth of $2^{\circ}$. Comparatively, the near field curves of ddm are almost parallel to horizontal axis and close to zero point. Corresponding to Figure 11, the value near the CL is poorly little.

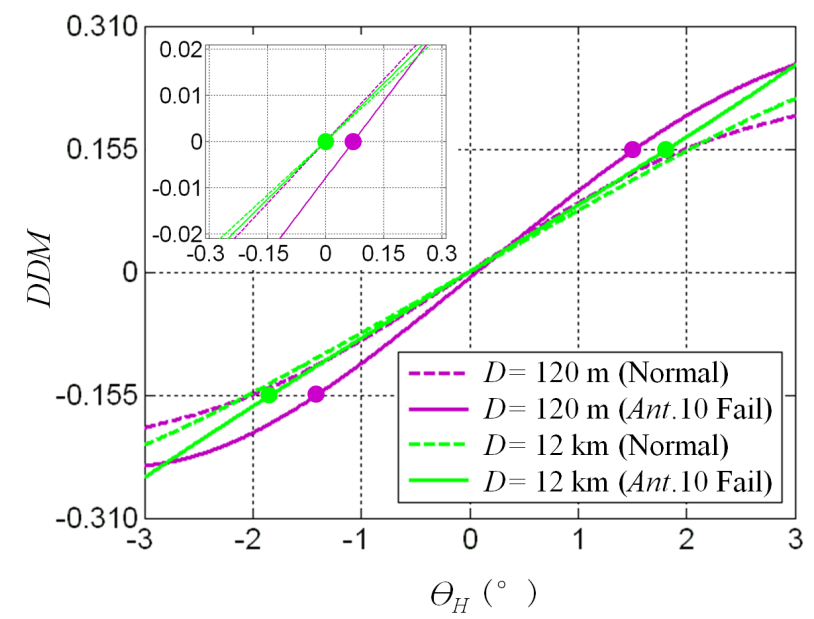

Figure 15. (Color Online) The horizontal azimuth dependence of ddm with $120 \mathrm{~m}$ for NF and $12 \mathrm{Km}$ for FF. The inset is the enlarged diagram, shows the situation near the CL. To compare the NF and FF circumstances more visual, the value of NF ddm modified up to 0.155 at $2^{\circ}$ with same DS to FF, seen the dotted lines. The solid lines are the situation for ant. 10 fail, no signal radiation. At far field, seen the green line, the DS turns to narrower. At near field, seen the purple line, the DS narrow down, but the extent is exceeding that of far field. Moreover, the CL deviates from the center line of run way, seen the inset. 


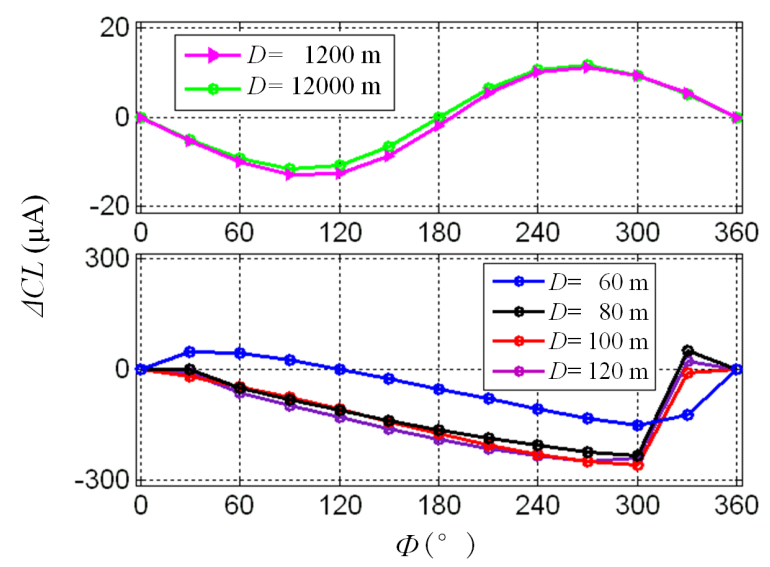

Figure 16. (Color Online) The phase calibration of shifter $\left(360^{\circ}\right.$ rotation) on Ant. 5 dependence of deviation of CL. For 12 $\mathrm{Km}$, seen the top one, when the phase increase, the value of $\triangle \mathrm{CL}$ become negative, the CL moves to the upper part of the center line of run way, seen the position of Figure 3. When the phase is increasing to $180^{\circ}$, the CL moves back and starts to moves to the other side the center line. Further moving to the starting point at $360^{\circ}$. The curve shows the odd symmetry behavior. For $1200 \mathrm{~m}$, the symmetry become a little worse, moving to right, at phase $180^{\circ}$, the value is negative. For near field situation, no symmetry could be found, while the extent of $\triangle \mathrm{CL}$ is amazing, about 10 times larger than that of far field.

perfect odd symmetry, the maximum variation $(\Delta \mathrm{CL})$ appears at $90^{\circ}$ and $270^{\circ}$, which is about $10 \mu \mathrm{A}$, and returns to zero at $180^{\circ}$. The latter one, less than $100 \mathrm{D}(2600 \mathrm{~m})$, has the almost the same maximum variation, but doesn't embody the good symmetry, the value deviates zero at $180^{\circ}$. The bottom one shows the NF result from $60 \mathrm{~m}$ to $120 \mathrm{~m}$, the symmetry disappeared completely. The curve of $60 \mathrm{~m}$ has a smaller variation, other three have the similar perform, exhibit negative value from $0^{\circ}-300^{\circ}$, the variation monotone increasing in this region, and exceeds $200 \mu \mathrm{A}$ at $300^{\circ}$. This variation is much more than that of FF. However, the situation in both NF and $\mathrm{FF}$, the variation is not is not so evident in the range from $\pm 30^{\circ}$, especially in NF.

\section{Summary}

Two models of Normarc localizer beacon 7220 A, far field model and near field models, were established and extensively studied by means of MATLAB modeling, theoretical analysis and experimental measuring. Far field model reflects infinite signal synthesis characteristic. The results are identical to that described in Normarc Training Manual. Near field model exhibits the situation that the receive distance is less than 100 times of the half aperture of antenna array. The distribution of signal coverage and DDM is extremely sensitive to the distance. So the NF model is more complex than FF one, and adds the order parameter: longitudinal dimension. This originates from the broken geometric symmetry in NF area. Accordingly, the NF plot pattern is significantly different from the plot of FF. The further simulation of antenna failed and phase shifter also confirmed this argument.

\section{References}

[1] Watts Jr., C.B. (2003) The Instrument Landing System: Replace It, or Repair It? The Journal of Navigation, 56, 411427.

[2] Normarc 7000B ILS (2013) Training Manual 24036-042.

[3] Normarc 3523B Instruction Manual (2011) 21450-39, Park Air Systems.

[4] Normarc 3524 Instruction Manual (2005) 21451-75, Park Air Systems.

[5] Normarc 3525 Instruction Manual (2011) 21452-0610, Park Air Systems.

[6] Normarc 3526 Instruction Manual (2011) 21453-32, Park Air Systems.

[7] Normarc 7212A Instruction Manual (2012) 23746-018, Park Air Systems.

[8] Normarc 7212C Instruction Manual (2012) 70375-012, Park Air Systems.

[9] Normarc 7216A Instruction Manual (2012) 24797-013, Park Air Systems.

[10] Normarc 7216C Instruction Manual (2012) 71507-01, Park Air Systems. 
[11] Normarc 7220A Instruction Manual (2012) 24136-015, Park Air Systems.

[12] Normarc 7220B Instruction Manual (2012) 24137-015, Park Air Systems.

[13] Thales ILS-LOC 411-Technical MANUAL (2000) Antenna Systems. Vol.1, Annes B.

[14] Butterworth, S. (1929) Note on the Apparent Demodulation of a Weak Station by a Stronger One. The Wireless Engineer and Experimental Wireless, VI.

[15] Annex 10 (2006) Aeronautical Telecommunications. Vol. I, 3.1.3.3.1, International Civil Aviation Organization.

[16] NORMARC 7710 User Manual (2006) 23327-Rev.1.4. Park Air Systems. 